Acta Crystallographica Section D

Biological

Crystallography

ISSN 0907-4449

Leila M. Foroughi, ${ }^{\text {a }}$ You-Na Kang $^{\mathrm{b}}$ and Adam J. Matzger ${ }^{\mathrm{a}, \mathrm{c} *}$

aDepartment of Chemistry, University of Michigan, Ann Arbor, MI 48109, USA,

b Life Sciences Institute, University of Michigan, Ann Arbor, MI 48109, USA, and ${ }^{\mathrm{c}}$ Department of Macromolecular Science and Engineering, University of Michigan, Ann Arbor, MI 48109, USA

Correspondence e-mail: matzger@umich.edu

\title{
Sixty years from discovery to solution: crystal structure of bovine liver catalase form III
}

The crystallization and structural characterization of bovine liver catalase (BLC) has been intensively studied for decades. Forms I and II of BLC have previously been fully characterized using single-crystal X-ray diffraction. Form III has previously been analyzed by electron microscopy, but owing to the thinness of this crystal form an X-ray crystal structure had not been determined. Here, the crystal structure of form III of BLC is presented in space group $P 2_{1} 2_{1} 2_{1}$, with unit-cell parameters $a=68.7, b=173.7, c=186.3 \AA$. The asymmetric unit is composed of the biological tetramer, which is packed in a tetrahedron motif with three other BLC tetramers. This higher resolution structure has allowed an assessment of the previously published electron-microscopy studies.

\section{Introduction}

Catalases are a class of heme proteins that catalyze the conversion of hydrogen peroxide to water and oxygen. In 1937, bovine liver catalase (BLC) was the first protein of this class to be crystallized (Sumner \& Dounce, 1937b). Sumner and Dounce employed several crystallization conditions that yielded multiple crystal morphologies. While additional protein-extraction and crystallization strategies were subsequently developed (Brown, 1952; Dounce, 1942; Mosimann, 1951; Tauber \& Petit, 1952), the conditions proposed by Sumner and Dounce became the most widely used method (McPherson \& Rich, 1973; Sumner \& Dounce, 1937a, 1955). The ability to readily purify BLC through crystallization allowed studies for the determination of the basic properties of the protein, including its molecular weight (Sumner \& Gralén, 1938) and number of biological subunits (Sumner \& Gralén, 1938; Valentine, 1964).

However, the studies mentioned above did not provide any insight into the crystal packing of the various forms. Electron microscopy (EM) provided the first insights into the unit-cell parameters and crystal packing of BLC. In an early study, Hall was able to determine the molecular weight and unit-cell parameters of a small needle-like morphology of BLC (Hall, 1950). Starting in the 1960s, BLC was commonly used in EM studies as both a calibration standard (Wrigley, 1968) and as the focus of experiments to determine the structure of various crystal forms (Dorset \& Parsons, 1975; Kiselev et al., 1967, 1968; Labaw, 1967; Massover, 1975; Matricardi et al., 1972; Taylor \& Glaeser, 1974; Unwin \& Henderson, 1975;
Received 27 May 2011

Accepted 22 June 2011

PDB Reference: bovine liver catalase, $3 \mathrm{nwl}$.
C 2011 International Union of Crystallography Printed in Singapore - all rights reserved 
Table 1

BLC forms structurally characterized by single-crystal X-ray diffraction.

\begin{tabular}{|c|c|c|c|}
\hline & Form I & Form II & Form III \\
\hline Space group & $P 3_{2} 21$ & $P 2_{1} 2_{1} 2_{1}$ & $P 2_{1} 2_{1} 2_{1}$ \\
\hline \multicolumn{4}{|l|}{ Unit-cell parameters } \\
\hline$a(\AA)$ & 142.0 & 87.8 & 68.7 \\
\hline$b(\AA)$ & 142.0 & 140.6 & 173.7 \\
\hline$c(\AA)$ & 103.7 & 232.4 & 186.3 \\
\hline$\alpha\left({ }^{\circ}\right)$ & 90 & 90 & 90 \\
\hline$\beta\left(^{\circ}\right)$ & 90 & 90 & 90 \\
\hline$\gamma\left({ }^{\circ}\right)$ & 120 & 90 & 90 \\
\hline Resolution $(\AA)$ & 2.5 & 2.3 & 2.7 \\
\hline PDB code & 7 cat & 4blc & $3 \mathrm{nwl}$ \\
\hline
\end{tabular}

Vainshtein et al., 1966, 1976; Valentine, 1964). The inherent concomitant growth of different forms of BLC crystals using the Sumner and Dounce conditions (Labaw, 1967; Sumner \& Dounce, 1937a, 1955; Sumner \& Gralén, 1938; Unwin, 1975), as well as the wide variety of crystal preparations that were used for EM studies (Akey \& Edelstein, 1983; Labaw, 1967; Unwin, 1975; Unwin \& Henderson, 1975), led to some confusion in the literature. Many papers suggested that all of the crystals used to perform EM studies were of the same form, even in cases where differences were observed in intensities. This led to doubt about the accuracy of the different packing models and proposed unit-cell parameters (Unwin, 1975). The confusion was then magnified by differences in reported space groups and unit-cell parameters, as well as the varying degrees of hydration of the crystals studied.

In the late 1960s, X-ray diffraction of BLC crystals began to be used both to verify the information gained by EM studies as well as to provide additional structural elucidation. The first X-ray experiments of BLC focused on prism-shaped crystals that had been identified in previous EM studies (Labaw, 1967) as belonging to an orthorhombic space group with unit-cell parameters $a=73, b=141, c=183 \AA$. After studying X-ray diffraction precession photographs, Rossmann and Labaw determined that these crystals were in fact of trigonal $P 3_{1} 21$ or $P 3_{2} 21$ form with unit-cell parameters $a=178.3, c=241.4 \AA$ (Labaw, 1967; Rossmann \& Labaw, 1967). This form was further studied by Longley (1967), who used both X-ray and EM diffraction patterns to determine the unit-cell parameters to be $a=173, c=237 \AA$ and $a=180, c=240 \AA$, respectively. The data obtained from the X-ray and EM experiments were combined to build a model of the packing at a resolution of $50 \AA$ A. Vainshtein, Gurskaya and coworkers worked extensively on the structural characterization of this form using $\mathrm{X}$-ray diffraction as well as studies that combined X-ray and EM data. These studies included the determination of unit-cell parameters using both X-ray diffraction and EM (Vainshtein et al., 1976), the creation of a low-resolution electron-density map using amplitudes from X-ray experiments and phase information calculated from EM data (Gurskaya et al., 1972) and the elucidation of the molecular symmetry of this form through a rotation-function study of $10 \AA$ data (Gurskaya, 1975). Most recently, the unit-cell parameters were again determined in an EM study at $20 \AA$ and computer-based reconstructions provided the most detailed crystal-packing information currently available for this form (Akey et al., 1984).

The second crystal form of BLC indexed using X-ray diffraction was hexagonal plates determined to belong to the orthorhombic space group $P 2_{1} 2_{1} 2_{1}$, with unit-cell parameters $a=140.9, b=231.2, c=87.2 \AA$ (Gurskaya et al., 1971). In 1973, the unit-cell parameters were again determined to be $a=89.1(5), b=140.0(5), c=231.2(20) \AA$ from X-ray precession photographs and a model was proposed based on the crystal packing observed by EM (McPherson \& Rich, 1973). Additionally, the unit-cell parameters of very thin plates of a second $P 2{ }_{1} 2_{1} 2_{1}$ form were determined to be $a=69$, $b=173.5, c=206 \AA$ through both EM and X-ray powder diffraction (Unwin, 1975). Finally, in 1976, a second distinct $P 3_{1} 21$ or $P 3_{2} 21$ trigonal form was discovered by Rossmann and Eventoff, with unit-cell parameters $a=b=142.3$ (6), $c=104.0$ (5) $\AA$ (Eventoff et al., 1976).

After these initial studies, BLC crystal forms continued to be analyzed extensively by X-ray and EM for several decades. The first solved crystal structure of BLC was for the second trigonal form (form I) in space group $P 3_{2} 21$, with unit-cell parameters $a=142.0, c=103.7 \AA$ (Table 1 ) at a resolution of $2.5 \AA$ (Murthy et al., 1981). This was followed by several papers that provided a further refined structure (Fita et al., 1986) and full elucidation of the heme (Reid et al., 1981) and NADPH (Fita \& Rossmann, 1985) binding sites. In 1999, the $\mathrm{X}$-ray single-crystal structure of the first orthorhombic $P 2_{1} 2_{1} 2_{1}$ form (form II), with unit-cell parameters $a=87.8, b=140.6$, $c=232.4 \AA$, at a resolution of $2.3 \AA$ was published (Ko et al., 1999).

The existence of the second $P 2_{1} 2_{1} 2_{1}$ crystal form (form III) was confirmed through attempts to further elucidate the unitcell parameters (Jésior, 1982) and packing of this form (Akey \& Edelstein, 1983; Unwin \& Henderson, 1975). One of the most thorough studies was Akey and Edelstein's projection of the crystal packing of form III along all three axes at a resolution of $20 \AA$ (Akey \& Edelstein, 1983). This provided the clearest projections of the packing of this form and illustrated that it was indeed a distinct crystal form of BLC. These results were validated by Dorset and Gilmore through their electroncrystallography studies of this crystal form at $9 \AA$ resolution (Dorset \& Gilmore, 1999). Additionally, this form has a long history of being used as a model for EM studies for the development of sample-preparation methods. Massover and coworkers used this form to develop non-heavy-metal negative-staining techniques for EM studies by soaking BLC in salts of sugars (Massover \& Marsh, 2000) and light-metal salts (Massover \& Marsh, 1997; Massover, 2008). Massover and coworkers also used BLC in studies to develop a screen for determining the proper sugars to use for structure preservation in EM studies (Massover et al., 2001; Massover, 2004). In addition, studies on this form of catalase have been used to help determine both the ideal temperature (Bammes et al., 2010; Brink et al., 1998) and exposure parameters (Baker et al., 2010) to minimize the signal-to-noise ratio while still maximizing the resolution. 
Form III of BLC has been extensively studied by EM and subsequently by electron crystallography as crystallization reproducibly provides plates that are less than $1 \mu \mathrm{m}$ thick (Unwin, 1975). This inherent thinness has made single-crystal $\mathrm{X}$-ray studies virtually impossible. Even with the progress in synchrotron beamlines which has allowed single-crystal X-ray diffraction of crystals with increasingly limited dimensions, a threshold crystal thickness of several micrometres is still required. This suggests that the ability to solve the singlecrystal structure of this form is dependent on implementing a suitable crystal-growth technique that will increase the crystal size. A heteronucleation technique based on insoluble polymers, polymer-induced heteronucleation, has successfully aided in crystal-form selection and discovery for a variety of small-molecule targets (López-Mejías et al., 2009; Lutker \& Matzger, 2010; Price et al., 2005; Porter et al., 2008; Roy \& Matzger, 2009). Recently, this method has been optimized and implemented for protein crystallization, including studying the crystallization of BLC (Foroughi et al., 2011). BLC crystals of form III grown on the polymers have an increased crystal size of $10 \mu \mathrm{m}$ in thickness, which allowed single-crystal X-ray diffraction and full structural elucidation for the first time. Here, we will describe the X-ray crystal structure of BLC form III.

\section{Materials and methods}

\subsection{Crystallization of BLC form III}

BLC was purchased from Sigma-Aldrich, St Louis, Missouri, USA (catalog No. C40). The condition used by McPherson and coworkers to crystallize form II of BLC (Ko et al., 1999), $40 \mathrm{mg} \mathrm{ml}^{-1}$ BLC in $0.05 \mathrm{M}$ sodium phosphate buffer $\mathrm{pH} 6.8$ and a precipitant solution consisting of $12 \%$ PEG 4000 and $0.05 M$ sodium phosphate buffer $\mathrm{pH} 6.8$, was used to obtain BLC forms I and II using the sitting-drop vapor-diffusion method. In the presence of a library of insoluble polymer heteronucleants, form III was also obtained from this condition (Foroughi et al., 2011). Form III crystals grown in the presence of PIHn showed a tenfold increase in crystal thickness compared with crystals grown in the absence of heteronucleants. One of these crystals with dimensions of $73 \times 15 \times$ $10 \mu \mathrm{m}$ was studied by single-crystal X-ray diffraction at Argonne National Laboratory. Full procedures for polymer preparation and the crystallization of BLC have been reported previously (Foroughi et al., 2011).

\subsection{Data collection}

Prior to data collection, BLC form III crystals were cryoprotected using a solution consisting of the mother liquor with $30 \%$ glycerol before freezing the crystals in liquid nitrogen. The X-ray diffraction data for BLC form III was collected at the Advanced Photon Source in Argonne National Laboratory on the LS-CAT beamline 21-ID-D using a MAR 300 detector and a wavelength of $1.127 \AA$ (Table 2). Data were collected using the standard oscillation method in $1.5^{\circ}$ increments with an exposure time of $1 \mathrm{~s}$ per image and a crystal-to-
Table 2

Diffraction data and structure-refinement summary for BLC form III.

Values in parentheses are for the highest resolution shell.

\begin{tabular}{ll}
\hline Data collection & \\
Beamline & 21 -ID-D, LS-CAT, APS \\
Space group & $P 2_{1} 2_{1} 2_{1}$ \\
Unit-cell parameters $(\AA)$ & 100 \\
Temperature $(\mathrm{K})$ & 1.127 \\
Wavelength $(\AA)$ & $50-2.69(2.76-2.69)$ \\
Resolution $(\AA)$ & $13.6(40.4)$ \\
$R_{\text {merge }}(\%)$ & $96.3(86.4)$ \\
Completeness $(\%)$ & $15.73(3.95)$ \\
$\langle I / \sigma(I)\rangle$ & $606094(23060)$ \\
Measured reflections & $60233(2672)$ \\
Unique reflections & $9.6(8.1)$ \\
Multiplicity & $3 n w 1$ \\
PDB entry & \\
Refinement & 57155 \\
No. of reflections & 20.4 \\
$R_{\text {work }}(\%)$ & 24.1 \\
$R_{\text {free }}(\%)$ & \\
No. of atoms per monomer & 1996 \\
Protein & 131 \\
Water & 364 \\
Ligands & \\
Average $B$ factors & 27.69 \\
Protein $\left(\AA^{2}\right)$ & $25.17,27.85,28.51,29.50$ \\
Chain $A, B, C, D\left(\AA^{2}\right)$ & 0.018 \\
R.m.s.d. bonds $(\AA)$ & 1.353 \\
R.m.s.d. angles $\left({ }^{\circ}\right)$ & $1.68[100$ th percentile] \\
MolProbity score & 97.54 \\
Ramachandran favored $(\%)$ & 0.10 \\
Ramachandran outliers $(\%)$ & \\
\hline
\end{tabular}

detector distance of $156 \mathrm{~mm}$. Diffraction data were processed using the HKL-2000 program package (Otwinowski \& Minor, 1997).

\subsection{Refinement}

The crystal structure of BLC form III was determined by molecular replacement using form II (PDB code 4blc; Ko et al., 1999) as a search model with the program Crystallography \& NMR System (CNS; Brünger et al., 1998). The structure was refined using restrained refinement using REFMAC5 (Murshudov et al., 2011) in the CCP4 software suite v.6.1.3 (Winn et al., 2011). A total of $5 \%$ of the reflections, which were randomly chosen and excluded from the refinement, were used for the calculation of $R_{\text {free }}$. Tight noncrystallographic symmetry (NCS) restraints were applied during the initial rounds of refinement and were gradually released in later rounds. Water molecules were added automatically and evaluated individually using Coot (Emsley \& Cowtan, 2004). Atomic coordinates and structure factors for BLC form III have been deposited in the PDB as entry 3nwl.

\section{Results}

\subsection{BLC form III crystal structure refinement}

The root-mean-square deviations (r.m.s.d.s) between the four monomers in the asymmetric unit were between 0.1 and $0.12 \AA$. An NADPH and a protoheme IX group were found in 
the active sites of each monomer. There were a total of 131 water molecules in the structure. The final $R$ and $R_{\text {free }}$ factors of the model were $20.4 \%$ and $24.1 \%$, respectively. The model was assessed with MolProbity (Chen et al., 2010; Table 2) and the Ramachandran plot showed that $97.54 \%$ of the main-chain dihedral angles lay in the most favored region, $2.36 \%$ lay in the additional favored region and $0.1 \%$ were outliers.

The average temperature factor over all 16553 atoms in the model was $27.69 \AA^{2}$. The average $B$ factors for chains $A, B, C$ and $D$ were $25.17,27.85,28.51$ and $29.50 \AA^{2}$, respectively. The average $B$ factors for the heme groups were 5.16, 5.61, 2.73 and $3.93 \AA^{2}$ and those for the NADPH group were $6.71,9.67,7.36$ and $6.79 \AA^{2}$ for chains $A, B, C$ and $D$, respectively (Table 2).

Even with the limitations in resolution, the electron density is well defined around both the NADPH and protoheme IX binding sites. The electron density around the heme is planar and it has hydrogen-bonding interactions with Arg364, Arg71 and Arg111. Additionally, Tyr357 coordinates with the iron in the heme group at a distance of $2 \AA$. There are water molecules distal to the heme pocket on the outer sides of the heme carboxylic acid chains for chains $A, C$ and $D$. In chain $B$ a water molecule is observed between the two carboxylic acid moieties and based on the electron density there may also be partial occupancy in the other chains. NADPH shares close contacts with His193, Ser200, Arg202, Lys236, Trp302 and His304.

\subsection{Structure of BLC form III}

The basic structure of the BLC monomers is unchanged from previously reported BLC structures (Ko et al., 1999; Reid et al., 1981): BLC is a tetramer consisting of four identical

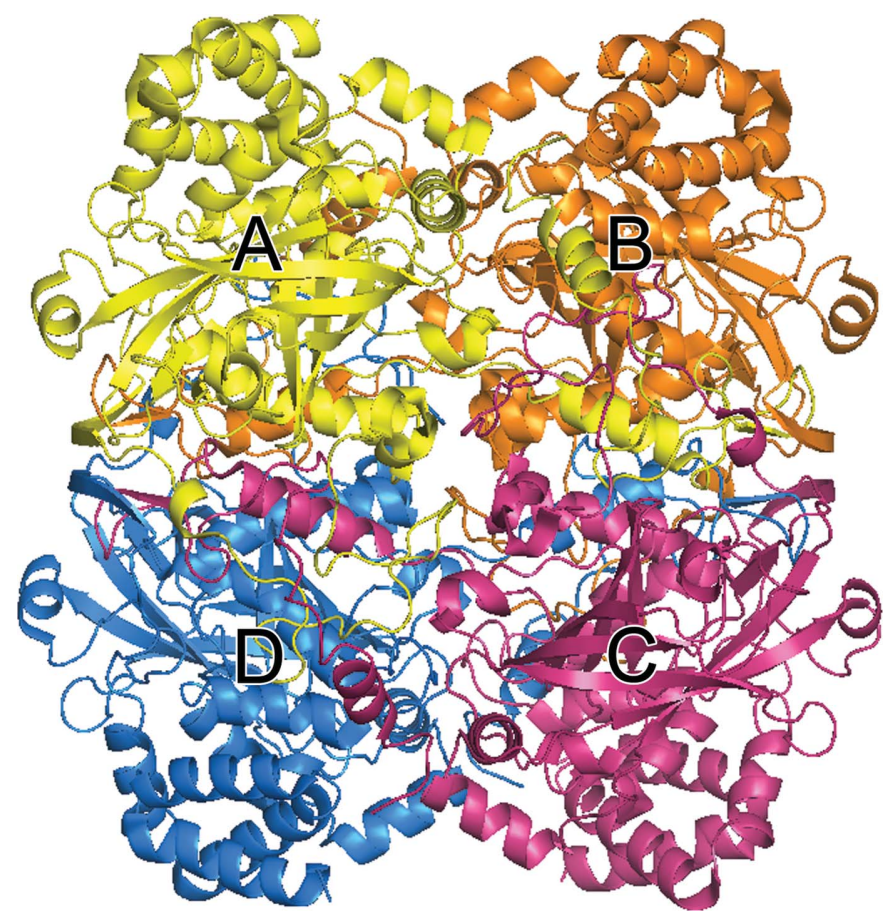

Figure 1

BLC tetramer consisting of four monomers $A, B, C$ and $D$. monomers, each with a molecular weight of $61 \mathrm{kDa}$. Each monomer contains two active sites, one that is iron-bound to a protoheme IX group and a second active site that can bind NADPH. The asymmetric unit for this structure is one biological tetramer with 222 point-group symmetry composed

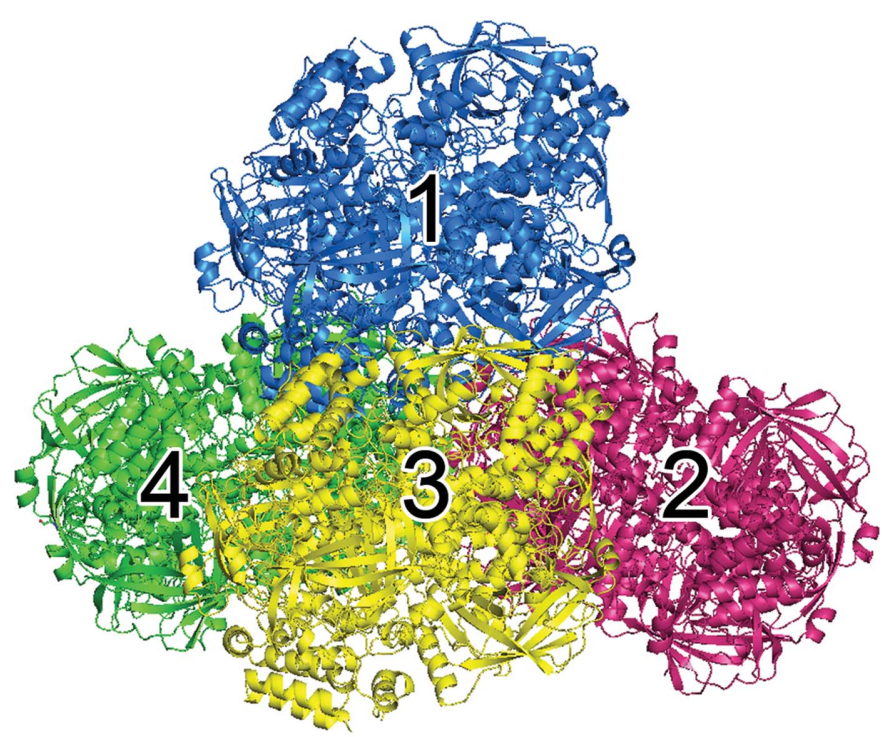

(a)

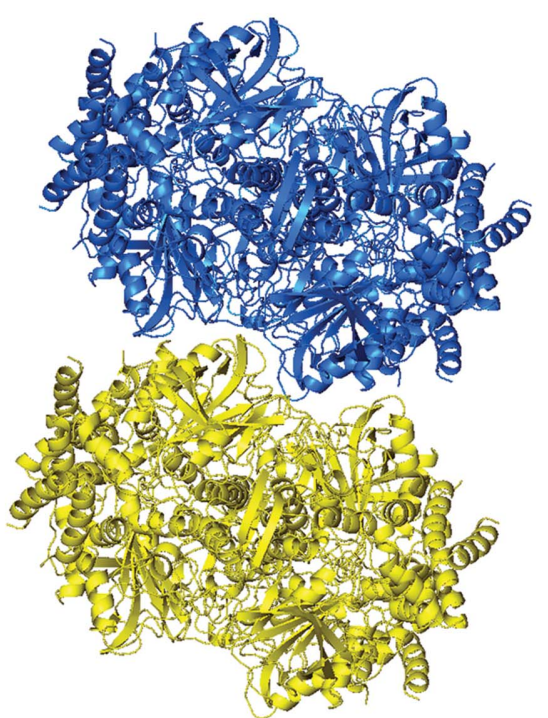

(b)

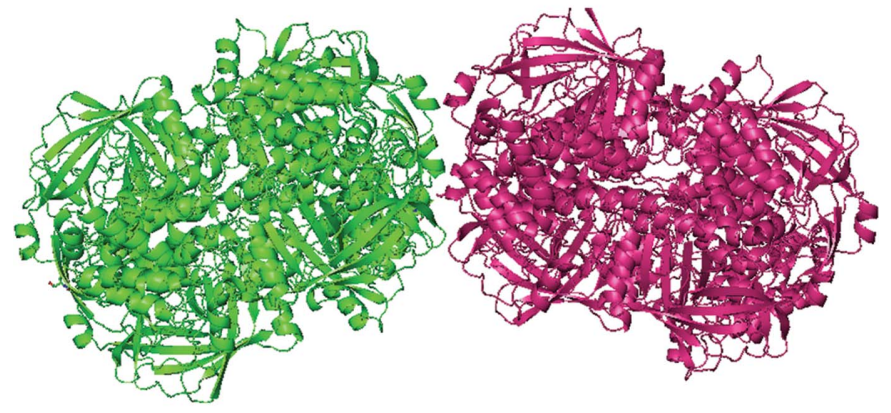

(c)

Figure 2

(a) Biological tetramer 1 packed in a tetrahedron motif with tetramers 2, 3 and 4. (b) Tetramers 1 and 3 and $(c)$ tetramers 2 and 4 make close contacts, including the hydrogen bonds and salt bridges listed in Table 3. 
of monomers $A-D$ (Fig. 1). The total surface area of the four chains is $108542 \AA^{2}$ and the surface area buried upon tetramerization is $53810 \AA^{2}$. As seen in Fig. 2(a), the tetramer is packed in a tetrahedron motif. Tetramers 1 and 3 (Fig. 2b) are packed parallel along the $a$ axis, with crystal contacts between $\operatorname{Arg} 105(A)$ and $\operatorname{Thr} 270(D), \operatorname{Arg} 379(A)$ and Gly271 $(D)$, Glu16 $(C)$ and $\operatorname{Arg} 381(D)$, and Asn3 $(C)$ and both Gln17 $(B)$ and Gln21 (B) (Table 3). Tetramers 2 and 4 (Fig. 2c) are closepacked by hydrogen-bonding crystal contacts between Ser 482 $(D)$ and Ser286 $(B)$ and between Ser286 $(C)$ and Ser482 $(A)$ as well as a salt bridge between Glu227 $(C)$ and Lys479 $(A)$.

Fig. 3 shows the crystal packing along all three crystal axes. When viewed along the $a$ axis, solvent channels are observed

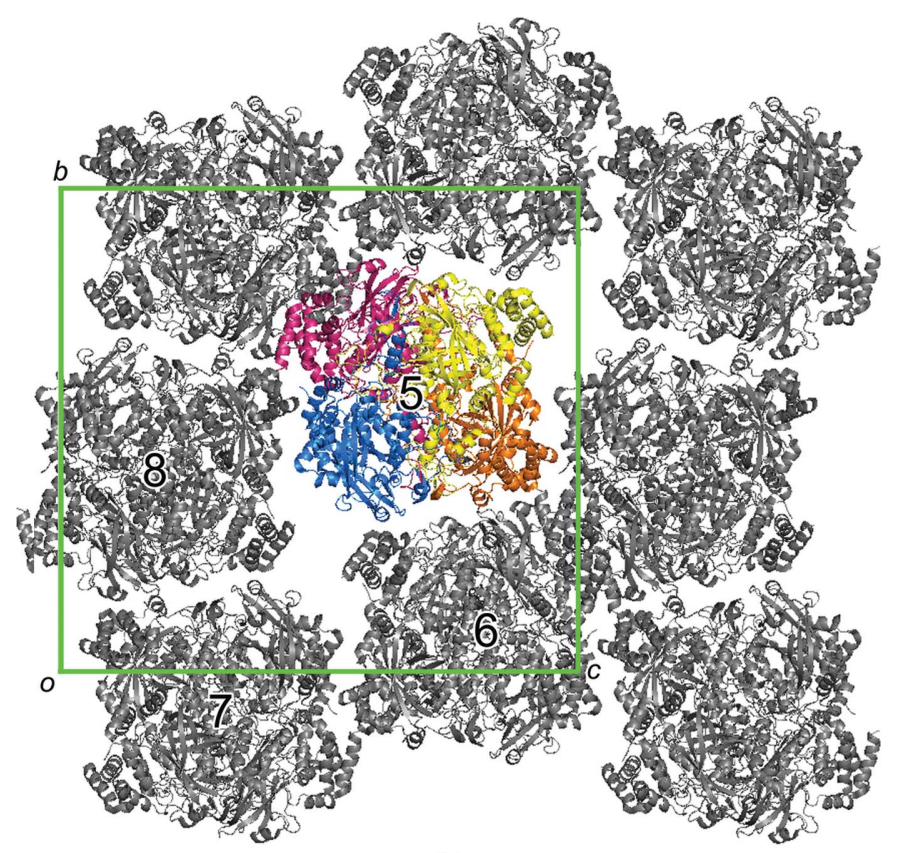

(a)

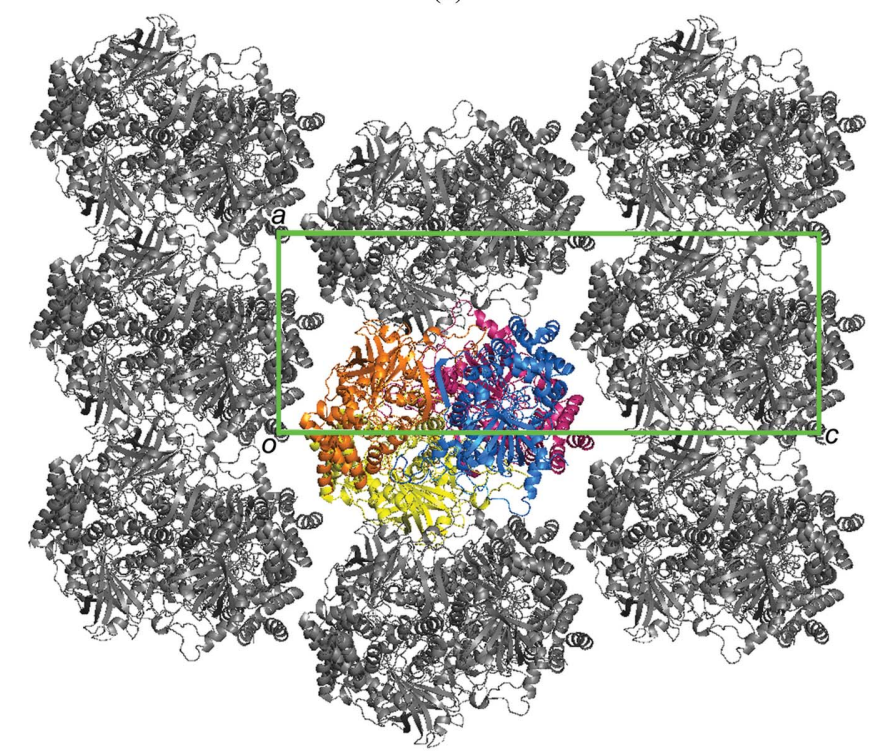

(b)
Table 3

BLC form III crystal contacts.

\begin{tabular}{llll}
\hline Residues & Tetramers & $\begin{array}{l}\text { Distance } \\
(\AA)\end{array}$ & Type \\
\hline Arg105 $(A)$ N-Thr270 $(D)$ NH2 & $1-3$ & 2.81 & Hydrogen bond \\
Arg379 $(A)$ NH2-Thr271 $(D)$ O & $1-3$ & 3.46 & Hydrogen bond \\
Arg379 $(C)$ NH1-Thr91 $(D)$ OG1 & $1-3$ & 3.67 & Hydrogen bond \\
Glu16 $(C)$ OE1-Arg381 $(D)$ NH2 & $1-3$ & 3.28 & Salt \\
Asn3 $(C)$ ND2-Gln17 $(B)$ O & $1-3$ & 3.25 & Hydrogen bond \\
Asn3 $(C)$ ND2-Gln21 $(B)$ OE1 & $1-3$ & 3.10 & Hydrogen bond \\
Ser482 $(C)$ O-Ser286 $(B)$ OG & $2-4$ & 2.73 & Hydrogen bond \\
Ser482 $(A)$ O-Ser286 $(C)$ OG & $2-4$ & 2.56 & Hydrogen bond \\
Lys479 $(A)$ NZ-Glu227 $(C)$ OE1 & $2-4$ & 3.84 & Salt \\
\hline
\end{tabular}

between four BLC tetramers (Fig. 3a). Tetramers 5 and 7 are related by a twofold rotation along the $a$ axis and the $32 \AA$ wide channel is flanked by $\alpha$-helices containing residues $486-$ 501. Tetramers 6 and 8 are also related by the same twofold rotation, with the channel being bordered by the $\alpha$-helices from residues $441-447$ and the loops from residues $289-305$ at a distance of $48.8 \AA$ apart.

\section{Discussion}

\subsection{Comparison of the crystal packing in BLC forms I, II and III}

The close-packing arrangement of BLC in form III can be compared with those previously observed for forms I and II. Trigonal form I is in space group $P 3_{2} 21$ and when viewed along the $c$ axis the two screw axes can be observed (Fig. $4 a$ ). In form II the biological tetramer (the asymmetric unit) is closepacked in a trigonal bipyramidal motif, which results in large channels (Fig. 4b) surrounded by six tetramers that have dimensions of $70.6 \times 85.4 \AA$ (Ko et al., 1999). The three forms have fairly similar solvent contents of $52.97,56.00$ and $49.97 \%$

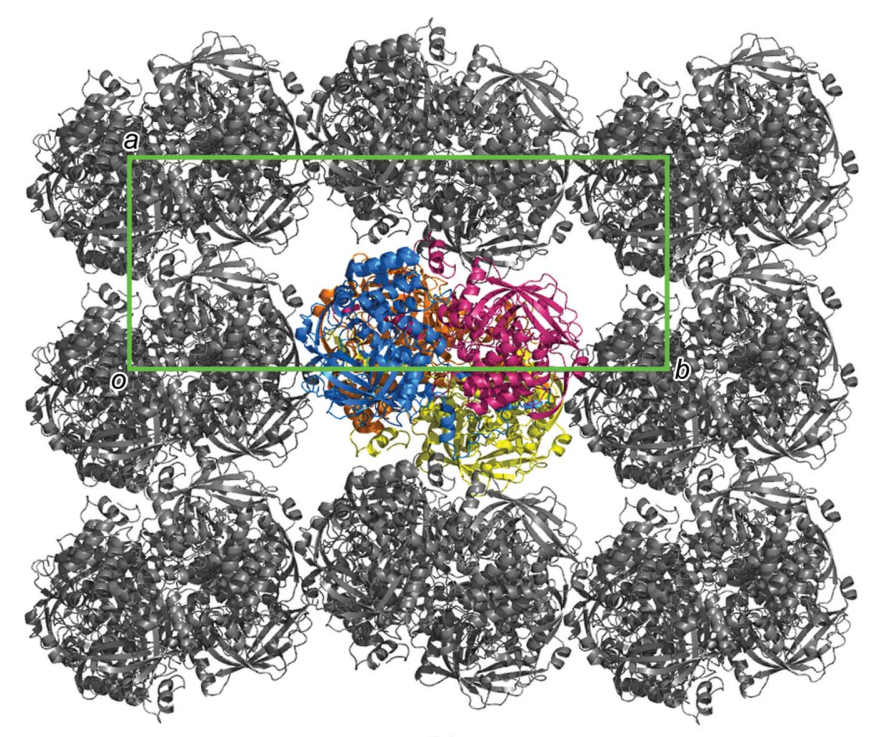

(c)

Figure 3

Crystal packing along the three axes. (a) Packing in the (100) plane; solvent channels are seen between four tetramers (tetramers 5-8). (b) Form III in the (010) plane. (c) Form III in the (001) plane. 
for forms I, II and III, respectively. The r.m.s.d.s between the different models of the tetramer are $0.33 \AA$ between forms I and III and $0.41 \AA$ between forms II and III.

\subsection{Comparison of the electron-microscopy data with the $\mathrm{X}$-ray single-crystal data}

The structural elucidation of form III is now complete and the previously collected electron-microscopy data can be compared with the X-ray crystal structure. EM data are most readily collected for the largest crystal face, the (001) face, which has allowed accurate measurements of the $a$ and $b$ axes. Unfortunately, determining the length of the $c$ axis has been challenging as it is by far the slowest growing direction, resulting in very thin crystals. In some instances, this form has only been identified based on the dimensions of the $a$ and $b$ axes (Dorset \& Parsons, 1975; Valentine, 1964; Wrigley, 1968). The most heavily referenced unit-cell parameters for form III are those from Unwin (1975): $a=69, b=173.5, c=206 \AA$ (Massover \& Marsh, 1997, 2000; Massover et al., 2001; Massover, 2004, 2008). The $c$ axis differs by about $10 \%$ from the dimensions published by Akey \& Edelstein (1983): $a=68$, $b=170, c=185 \AA$ A Since the crystal packing proposed for both these forms is very similar, it was suggested that the difference was a consequence of different growth conditions leading to different degrees of hydration. These latter dimensions have now been confirmed by the X-ray crystal structure with unitcell parameters $a=68.7, b=173.7, c=186.3 \AA$.
Although the EM studies were useful in providing unit-cell parameters and packing for the various forms, there are still inherent limitations to what can be observed when molecules can only be viewed down the crystal faces. This led to confusion in calculating the BLC tetramer size and shape, which was suspected to be perhaps cuboidal, spherical or ellipsoidal (Dorset \& Parsons, 1975; Hall, 1950; Valentine, 1959, 1964). Some studies gave a possible protein width of between 70 and $80 \AA$ (McPherson \& Rich, 1973; Rossmann \& Labaw, 1967). In 1959 , Valentine suggested that anhydrous BLC had a diameter of $84 \AA$, with a length:width ratio of 3:4 (Valentine, 1959). Gurskaya and coworkers suggested dimensions of $70 \times 90 \times$ $100 \AA$ from X-ray and EM studies (Gurskaya et al., 1972). Additionally, Akey and Edelstein measured the tetramer dimensions to be $89 \times 68 \times 90 \AA$ for form III of BLC (Akey \& Edelstein, 1983). These dimensions roughly agree with the dimensions measured from the X-ray crystal structure of this form when observing the molecule along the three axes. However, as is the case in other BLC forms, the molecules are tilted and not in their fully upright position along the axes and relying solely on data from a two-dimensional vantage point can lead to inaccuracies in molecule size. The actual size of the tetramer is $105 \times 60 \times 105 \AA$.

\subsection{Insight into BLC form III crystal morphology}

As stated above, BLC form III consistently grows as thin plates with a thickness of less than $1 \mu \mathrm{m}$ (Unwin, 1975); however, the theory of Bravais, Friedel, Donnay and Harker

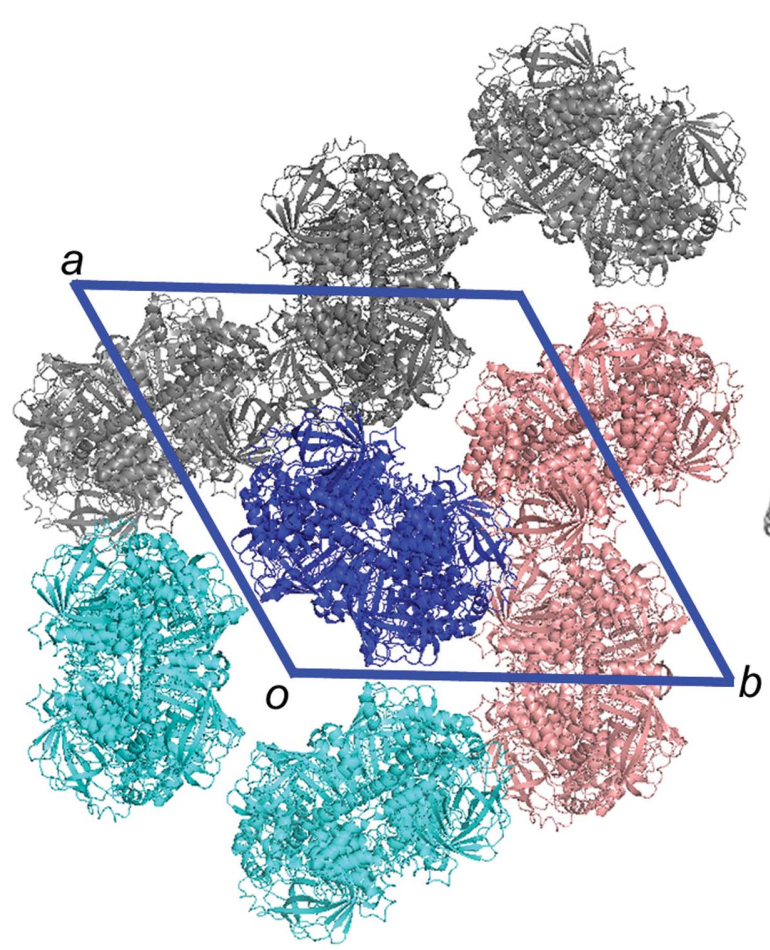

(a)

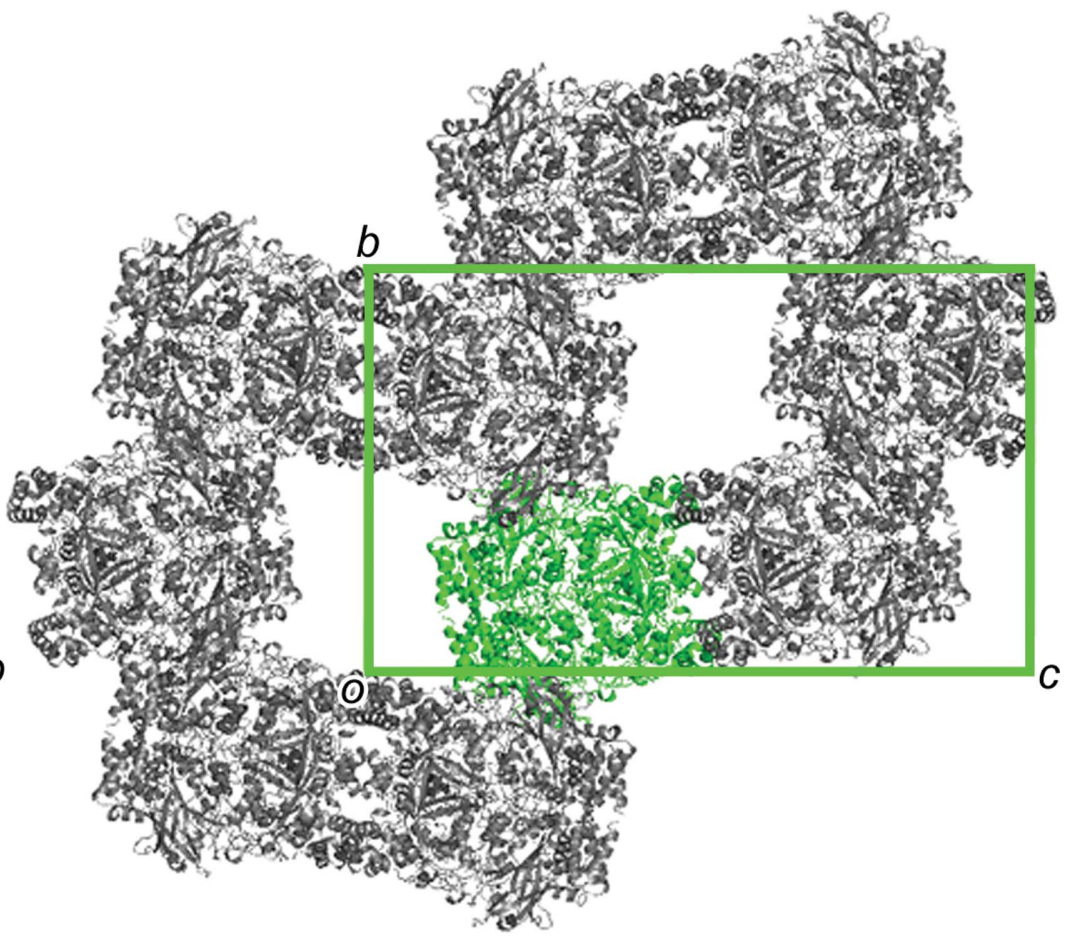

(b)

Figure 4

Crystal packing of BLC forms I ( $a$ ) and II ( $b$ ) viewed along the $c$ and $a$ axes, respectively. ( $a$ ) The dark blue tetramer forms a solvent channel with the two cyan tetramers along one screw axis. The two salmon tetramers share hydrogen-bonding interactions with the dark blue tetramer along the second screw axis. (b) The green BLC tetramer forms large solvent channels with six BLC monomers. 
(Donnay \& Harker, 1937) predicts that the morphology of these crystals will be blocky. Looking to bridge this disconnect between the predicted and the observed morphology, insight can be gained by analyzing the interactions between protein molecules. None of the close crystal contacts that are observed in form III are along the thin $c$ axis, suggesting a poor thermodynamic driving force for assembly in this direction. Although extreme anisotropy is not observed in the X-ray diffraction patterns; the highest $B$ factors are observed for residues exposed along the $c$ axis. This is particularly true for chain $A$, where the $B$ factors for Arg491, Asp497, Lys498, Tyr499, Asn500 and Glu501 are 93.0, 83.4, 89.9, 81.0, 85.7 and $101.0 \AA^{2}$, respectively. The form III crystals grown using the polymer-induced heteronucleation crystallization method were thicker than those grown using conventional methods. This may result from the induction of crystal growth at relative low extents of supersaturation, thus promoting a more equilibriumlike morphology and resulting in thicker crystals.

Use of the Advanced Photon Source was supported by the US Department of Energy, Office of Science, Office of Basic Energy Sciences under Contract No. DE-AC02-06CH11357. Use of the LS-CAT Sector 21 was supported by the Michigan Economic Development Corporation and the Michigan Technology Tri-Corridor for the support of this research program (grant 085P1000817). This study received funding through the Center for Chemical Genomics Pilot Project Initiative at the University of Michigan sponsored by Thermo Fisher Scientific.

\section{References}

Akey, C. W. \& Edelstein, S. J. (1983). J. Mol. Biol. 163, 575-612.

Akey, C. W., Szalay, M. \& Edelstein, S. J. (1984). Ultramicroscopy, 13, 103-111.

Baker, L. A., Smith, E. A., Bueler, S. A. \& Rubinstein, J. L. (2010). J. Struct. Biol. 169, 431-437.

Bammes, B. E., Jakana, J., Schmid, M. F. \& Chiu, W. (2010). J. Struct. Biol. 169, 331-341.

Brink, J., Sherman, M. B., Berriman, J. \& Chiu, W. (1998). Ultramicroscopy, 72, 41-52.

Brown, G. L. (1952). Biochem. J. 51, 569-573.

Brünger, A. T., Adams, P. D., Clore, G. M., DeLano, W. L., Gros, P., Grosse-Kunstleve, R. W., Jiang, J.-S., Kuszewski, J., Nilges, M., Pannu, N. S., Read, R. J., Rice, L. M., Simonson, T. \& Warren, G. L. (1998). Acta Cryst. D54, 905-921.

Chen, V. B., Arendall, W. B., Headd, J. J., Keedy, D. A., Immormino, R. M., Kapral, G. J., Murray, L. W., Richardson, J. S. \& Richardson, D. C. (2010). Acta Cryst. D66, 12-21.

Donnay, J. D. H. \& Harker, D. (1937). Am. Miner. 22, 446-467.

Dorset, D. L. \& Gilmore, C. J. (1999). Acta Cryst. A55, 448-456.

Dorset, D. L. \& Parsons, D. F. (1975). Acta Cryst. A31, 210-215.

Dounce, A. L. (1942). J. Biol. Chem. 143, 497-501.

Emsley, P. \& Cowtan, K. (2004). Acta Cryst. D60, 2126-2132.

Eventoff, W., Tanaka, N. \& Rossmann, M. G. (1976). J. Mol. Biol. 103, 799-801.

Fita, I. \& Rossmann, M. G. (1985). Proc. Natl Acad. Sci. USA, 82, 1604-1608.
Fita, I., Silva, A. M., Murthy, M. R. N. \& Rossmann, M. G. (1986). Acta Cryst. B42, 497-515.

Foroughi, L. M., Kang, Y.-N. \& Matzger, A. J. (2011). Cryst. Growth Des. 11, 1294-1298.

Gurskaya, G. V. (1975). Kristallografiya, 20, 516-523.

Gurskaya, G. V., Karpukhi, S. Y. \& Lobanova, G. M. (1971). Biophysics, 16, 576.

Gurskaya, G. V., Vainshtein, B. K. \& Lobanova, G. M. (1972). Sov. Phys. Crystallogr. 16, 662-669.

Hall, C. E. (1950). J. Biol. Chem. 185, 749-754.

Jésior, J. C. (1982). EMBO J. 1, 1423-1428.

Kiselev, N. A., De Rosier, D. J. \& Klug, A. (1968). J. Mol. Biol. 35, 561-566.

Kiselev, N. A., Shpitzberg, C. L. \& Vainshtein, B. K. (1967). J. Mol. Biol. 25, 433-441.

Ko, T.-P., Day, J., Malkin, A. J. \& McPherson, A. (1999). Acta Cryst. D55, 1383-1394.

Labaw, L. W. (1967). J. Ultrastruct. Res. 17, 327-341.

Longley, W. (1967). J. Mol. Biol. 30, 323-327.

López-Mejías, V., Kampf, J. W. \& Matzger, A. J. (2009). J. Am. Chem. Soc. 131, 4554-4555.

Lutker, K. M. \& Matzger, A. J. (2010). J. Pharm. Sci. 99, 794-803.

Massover, W. H. (1975). Biophys. J. 15, A326.

Massover, W. H. (2004). Microsc. Microanal. 10, 261-269.

Massover, W. H. (2008). Microsc. Microanal. 14, 126-137.

Massover, W. H., Lai, P. F. \& Marsh, P. (2001). Ultramicroscopy, 90, 7-12.

Massover, W. H. \& Marsh, P. (1997). Ultramicroscopy, 69, 139-150.

Massover, W. H. \& Marsh, P. (2000). Ultramicroscopy, 85, 107-121.

Matricardi, V. R., Moretz, R. C. \& Parsons, D. F. (1972). Science, 177, 268-270.

McPherson, A. \& Rich, A. (1973). Arch. Biochem. Biophys. 157, 23-27.

Mosimann, W. (1951). Arch. Biochem. Biophys. 33, 487-488.

Murshudov, G. N., Skubák, P., Lebedev, A. A., Pannu, N. S., Steiner, R. A., Nicholls, R. A., Winn, M. D., Long, F. \& Vagin, A. A. (2011). Acta Cryst. D67, 355-367.

Murthy, M. R., Reid, T. J., Sicignano, A., Tanaka, N. \& Rossmann, M. G. (1981). J. Mol. Biol. 152, 465-499.

Otwinowski, Z. \& Minor, W. (1997). Methods Enzymol. 276, 307-326.

Porter, W. W., Elie, S. C. \& Matzger, A. J. (2008). Cryst. Growth Des. 8, 14-16.

Price, C. P., Grzesiak, A. L. \& Matzger, A. J. (2005). J. Am. Chem. Soc. 127, 5512-5517.

Reid, T. J., Murthy, M. R., Sicignano, A., Tanaka, N., Musick, W. D. \& Rossmann, M. G. (1981). Proc. Natl Acad. Sci. USA, 78, 4767-4771.

Rossmann, M. G. \& Labaw, L. W. (1967). J. Mol. Biol. 29, 315-316.

Roy, S. \& Matzger, A. J. (2009). Angew. Chem. Int. Ed. 48, 8505-8508.

Sumner, J. B. \& Dounce, A. L. (1937a). J. Biol. Chem. 121, 417-424.

Sumner, J. B. \& Dounce, A. L. (1937b). Science, 85, 366-367.

Sumner, J. B. \& Dounce, A. L. (1955). Methods Enzymol. 2, 775-781.

Sumner, J. B. \& Gralén, N. (1938). Science, 87, 284.

Tauber, H. \& Petit, E. L. (1952). J. Biol. Chem. 195, 703-706.

Taylor, K. A. \& Glaeser, R. M. (1974). Science, 186, 1036-1037.

Unwin, P. N. (1975). J. Mol. Biol. 98, 235-242.

Unwin, P. N. \& Henderson, R. (1975). J. Mol. Biol. 94, 425-440.

Vainshtein, B. K., Kiselev, N. A. \& Shpitsberg, V. L. (1966). Dokl. Akad. Nauk SSSR, 167, 212-214.

Vainshtein, B. K., Sherman, M. B. \& Barynin, V. V. (1976). Kristallografiya, 21, 520-523.

Valentine, R. C. (1959). Nature (London), 184, 1838-1841.

Valentine, R. C. (1964). Nature (London), 204, 1262-1264.

Winn, M. D. et al. (2011). Acta Cryst. D67, 235-242.

Wrigley, N. G. (1968). J. Ultrastruct. Res. 24, 454-464. 